\title{
AMBIVALENCE MATERNELLE
}

\section{Michèle Benhaïm}

\author{
ERES | « Spirale »
}

2002/1 n $\mathrm{n}^{0} 21 \mid$ pages 99 à 107

ISSN 1278-4699

ISBN 2749200288

\section{Article disponible en ligne à l'adresse :}

http://www.cairn.info/revue-spirale-2002-1-page-99.htm

\section{Pour citer cet article :}

Michèle Benhaïm, «Ambivalence maternelle », Spirale 2002/1 (nº 21), p. 99-107. DOI 10.3917/spi.021.0099

Distribution électronique Cairn.info pour ERES.

(C) ERES. Tous droits réservés pour tous pays.

La reproduction ou représentation de cet article, notamment par photocopie, n'est autorisée que dans les limites des conditions générales d'utilisation du site ou, le cas échéant, des conditions générales de la licence souscrite par votre établissement. Toute autre reproduction ou représentation, en tout ou partie, sous quelque forme et de quelque manière que ce soit, est interdite sauf accord préalable et écrit de l'éditeur, en dehors des cas prévus par la législation en vigueur en France. Il est précisé que son stockage dans une base de données est également interdit. 


\section{Michèle Benhaïm}

\section{Ambivalence maternelle}

"C'est dans la dialectique de la demande d'amour et dans l'épreuve du désir, que le développement sordonne." J. Lacan, Écrits, p. 693

Winnicott considérait que la réussite de la fonction maternelle s'incarnait dans « une mère suffisamment bonne ». C'est ce point un peu énigmatique que $\mathrm{j}$ 'interroge depuis une quinzaine d'années, et notamment aujourd'hui autour de la question de l'ambivalence de la mère. Deux remarques me semblent importantes à proposer d'emblée.

La première concerne la traduction approximative du good enough mother; en effet, si je préfere pour ma part la " juste assez mais pas trop ", c'est parce que good enough se traduit en réalité par " insuffisamment bonne mais je m'en suis contenté ». Cette ambiguïté contient cependant d'ores et déjà le mouvement ambivalent qui va nous occuper, et a l'intérêt de souligner que le enough signifiant tout autant "suffisamment " et "assez ", indique à la fois une satisfaction et l'atteinte d'un seuil à ne pas dépasser. Ce qui, en matière d'amour maternel n'est pas anodin.

La deuxième remarque concerne ce qui, dans la fonction maternelle, relève déjà d'un champ symbolique comme condition, comme support, comme autorisant que la métaphore

Michèle Benhä̈m, psychanalyste, psychologue clinicienne en psychiatrie (CHS Valvert) et à Médecins du monde (Marseille). 
paternelle opère. À l'intérieur de ce champ déjà symbolique se construit une humanisation de base (au travers notamment des soins corporels et du nourrissage). Seule cette relation humanisante peut éclairer la place symbolique de l'enfant, temps où la mère porte et tisse le lien entre le corps et l'environnement.

Parler d'ambivalence, c'est se situer dans la dimension structurelle de ce concept. C'est la structure ambivalente de la mère qui importe et non, bien sûr, son comportement, au sens où l'on peut dire communément que quelqu'un est ambivalent parce qu'il ne sait pas ce qu'il veut. Ici, ce qui structurerait la mère, c'est l'ambivalence au sens où le sentiment maternel n'est pas univoque et qu'il y a bien un "au-delà de l'amour maternel». Cet au-delà, on le situera du côté de la haine, non pas comme un sentiment qui annulerait l'amour, mais comme un sentiment qui le traverserait. Il ne s'agit pas ici d'assimiler la haine à son aspect destructeur mais à l'autre face de l'amour au sens où l'on peut dire par exemple du transfert dans la cure qu'il est négatif, ce qui n'empêche pas la relation transférentielle de demeurer dans le registre de l'amour. Ainsi, la haine, ici, c'est ce qui limite l'amour au sens d'une limite de la jouissance maternelle qui, si elle garde l'aspect d'un amour sans limite, amour inconditionnel, risque de se renverser pour le coup en haine destructrice, voire mortelle. L'amour inconditionnel étant celui qui fait l'économie psychique de la castration, cela nous donne d'ores et déjà une indication quant au rapport que la mère entretenait comme femme, avec le manque.

L'ambivalence maternelle, si on ne la considère donc pas comme une formation pathologique mais au contraire comme la condition même de la coupure symbolique mère/enfant, réconcilie la mère réelle, celle de la toute-puissance, la mère de la première tétée, celle dont l'autiste par exemple ne saurait se détacher, la mère imaginaire, celle du miroir et de la reconnaissance de l'enfant que ce stade fonde, enfin la mère symbolique, agent d'une frustration signifiante.

Au sein de ces trois imagos maternelles réunifiées, peut se mettre en mouvement le processus d'individuation-séparation : individuation de l'enfant et séparation d'avec la mère reposant sur l'aptitude de celle-ci à accepter ses propres sentiments de haine à l'égard de son enfant, condition pour qu'elle ne se précipite pas dans l'agir, pouvant aller jusqu'à l'infanticide. 
Le constat clinique qui illustre cette hypothèse est celui de la mère qui, quittant une position quasi psychotique de " préoccupation maternelle primaire ", hésitera avant de penser le cri de l'enfant : pleure-t-il parce qu'il a faim, froid, peur... ? La trop bonne mère répondra immédiatement, ne laissant ainsi aucun écart entre demande et satisfaction du besoin, c'est-à-dire aucune place au désir ; la mère trop haineuse ne répondra que par un rejet, parfois violent. La mère ordinaire, « juste assez mais pas trop ", mettra son ambivalence au service d'une interprétation du cri de l'enfant. Interprétation elle-même déjà marquée de la propre castration maternelle - on n'est jamais comblé, on est seulement en partie satisfait - et participant de la sorte à l'humanisation de l'enfant.

La mère ambivalente met fin aux certitudes maternelles en conjuguant hésitation et engagement. À l'inverse, dans le champ. des excès, on se heurte à une clinique du " caprice " maternel qui fait de la production d'un corps le support réel de ce qui ne doit être d'emblée que symbolique. Autrement dit une clinique qui témoigne de mères demeurant dans la croyance qu'avoir un enfant c'est le posséder, à l'endroit où lorsqu'une femme énonce "j'ai un enfant ", c'est que précisément elle ne l'a plus.

Ce point nous amène à évoquer la place que l'enfant vient occuper dans le fantasme maternel et c'est, me semble-t-il, le point central du travail avec un parent, avec une mère d'enfant malade par exemple, celui auquel la mère se dévoue toute, travail d'élaboration articulé autour de la question de savoir en quoi l'enfant peut faire symptôme dans la relation à sa mère. Symptôme au sens de compromis permettant de soutenir une structure de désir, en l'occurrence ici le désir de la mère dans lequel l'enfant s'inscrit toujours, se départit au mieux, reste aliéné au pire.

L'ambivalence nous fait situer le point d'origine de la haine contenue et limitant l'amour du côté de la mère et non, comme on a coutume de le penser, du côté de l'enfant. Haine originaire qui se résout en général en amour maternel divisé, pas tout, c'est-à-dire ni comblé ni comblant. Derrière l'impossibilité d'une telle symbolisation, l'analyse de la mère mène à un écueil dans l'élaboration de sa position narcissique. Je rappelle ici que le mythe de Narcisse fait référence à une demande d'amour jamais satisfaite. Freud y faisait reposer sa Névrose narcissique, que nous nommons aujourd'hui psychose, illustrant comment le 
sujet pris dans l'impossibilité de trouver une réponse à l'extérieur rompt les échanges avec cet extérieur et retourne ses énergies pulsionnelles à l'intérieur.

Nous articulons pour notre part, et à sa suite, cet écueil à un rapport particulier à la castration qui voit dans la naissance d'un enfant se superposer un deuil infaisable (celui de la position narcissique maternelle) et une perte inintégrable (celle de l'enfant qui, dès lors qu'il paraît, se doit d'être un autre radical).

L'enfant, une fois là, ne cesse d'interroger le désir de sa mère. La symbolisation est le reflet des mots et des fantasmes maternels qui portent l'enfant et l'accueillent. Qu'aucun objet ne puisse prétendre combler le manque de la mère, pas même un enfant, doit être intégré par elle pour que l'enfant, objet imaginaire, puisse être " perdu ", autrement dit pour qu'elle puisse s'en séparer. À défaut, il peut prendre le sens d'un objet " réel ", et ce statut le confine au creux d'une mère alors totalement dévouée à cet objet. Les signes précurseurs de ce travail de séparation se repèrent cliniquement par exemple dans cet état dépressif qui suit l'accouchement et qui inaugure ce travail d'élaboration de la perte.

Finalement, il s'agit, en donnant la vie, de faire le deuil non seulement de l'enfant porté mais également de celui qui vient de naître. Pourquoi est-ce si difficile d'en finir avec le miroir de la fusion, avec l'illusion d'un " corps dans corps ", avec le fantasme de faire du un avec du deux ? Sans doute parce que la grossesse suppose que, pour un temps, le principe de plaisir prenne l'avantage sur le principe de réalité. Ainsi peut-elle au mieux relever d'un enchantement qui, contenant sa propre limite, verra la rupture inhérente à la mise au monde relever d'une double perte : celle de cet état d'enchantement (superposition singulière et exceptionnelle de l'Imaginaire et du Réel) et celle de l'objet (l'enfant). Au pire elle touchera au ravissement, voire au ravage, ce lien dont on ne peut venir à bout. Lien reposant sur un défaut de reconnaissance d'élaboration de ces ruptures et qui peut empêcher que la mère établisse des liens entre cet enfant qu'elle a en elle, celui qu'elle est, celui qu'elle a porté, celui qui est apparu et enfin celui dont elle se préoccupe. Il s'agit ici de reconnaître un compromis à négocier entre les exigences du fantasme et celles de la réalité. On peut supposer qu'un enfant porté neuf mois ait revêtu des aspects d'objet intérieur essentiel pour le corps de la mère. Les investissements de cette dernière doivent 
passer de cet objet intérieur qui favorisait un certain repli narcissique, une certaine gratification narcissique, à un objet extérieur nouveau. Pour désinvestir l'enfant sur ce mode narcissique primaire (temps de l'unité), l'introduction d'un troisième est d'ores et déjà inexorable. Ainsi, c'est dès le début que l'enfant rencontre les objets du désir de sa mère.

La première relation objectale avec l'enfant une fois établie, sur un mode régressif nécessairement induit par la préoccupation maternelle primaire, se fonde donc sur le modèle œedipien dans lequel s'est nouée la première relation de la mère avec sa propre mère. L'analyse nous révèle que ce chemin est à chaque fois singulier. Moins la mère "sait " le désir de l'enfant, plus il émerge comme sujet désirant. Ce passage de l'interprétation du cri à l'écoute d'une parole recouvre le passage de l'imaginaire au symbolique, d'une relation prégénitale à une relation génitale, et correspond à ce qui, du corps à corps, s'efface pour laisser place au corps autonome. Passage du fantasme au mythe que seule autorise la métaphore paternelle qui vient signifier à l'enfant qu'il n'est pas le premier signifiant de la chaîne, qu'il transcende la subjectivité de la mère, autrement dit qu'il est un maillon dans une filiation et qu'il est né d'une alliance. La question de l'ambivalence maternelle n'aurait pas de raison d'être " hors sujet ". Et, à l'origine du sujet est la métaphore. C'est pourquoi "s'il n'est pas nécessaire qu'il y ait un homme pour qu'il y ait un père » (Joël Dor), ajoutons cependant qu'il ne saurait y avoir de mère sans père. Père ni incarné ni géniteur, mais pouvant supporter une signifiance symbolique au regard du désir de l'enfant en prise avec le désir de la mère.

$\mathrm{Si}$, en introduisant mon propos, je mettais l'accent sur ce que la fonction maternelle contenait de déjà symbolique, c'est parce que la métaphore du Nom du Père ne sera opérante quà la condition que soit surmontée l'épreuve de la séparation, tout en étant nécessaire à son dépassement. Autrement dit que le désir de la mère soit lié à cette loi qui fait d'une relation dyadique ou fusionnelle avec l'enfant une relation d'ores et déjà œdipienne.

$\mathrm{Au}$ sein même de la préoccupation maternelle primaire qui voyait l'enfant saisi dans une modalité relationnelle à la mère où le père réel serait vécu comme "étranger ", si imaginairement l'enfant semblait combler la mère et être en retour comblé par elle, cette dernière était d'emblée " suffisamment bonne " pour 
entretenir le leurre en deçà d'une certaine limite, et néanmoins déjà " suffisamment haineuse " pour n'en être pas dupe, c'est-àdire pour autoriser, au-delà de cette limite, l'interruption du rêve auquel, sinon, se substituerait le délire. Personne n'a comblé personne puisque l'un peut survivre à l'absence de l'autre. La mère a donc encore à rencontrer sexuellement un père, et l'enfant ainsi autrement investi peut établir de nouvelles relations objectales.

L'ambivalence permet ce changement de position subjective maternelle comme en écho à un changement de statut de l'enfant, passages s'élaborant l'un comme l'autre, l'un avec l'autre, l'un par l'autre, sur une scène nouvelle où pour être deux il faut être trois.

L'unité narcissique primaire (Philippe Gutton) a laissé place à une organisation déjà œedipienne. L'enfant érotiquement désinvesti remet la mère face à son désir, désir qui ne saurait le concerner plus avant.

Être une mère ambivalente pourrait consister en partie à dissocier les registres du sexuel et du maternel à l'endroit de l'enfant. Envisagée de la sorte, on peut admettre que la haine comme envers de l'amour et qui fonde cette ambivalence, ne relève pas d'une pathologie relationnelle mais conditionne nécessairement et de façon structurante la séparation mère/enfant, coupure symbolique qui voit l'une réinvestir le champ du féminin, et l'autre partir vivre sa vie sans trop de remords.

C'est dans la brèche qu'ouvre l'ambivalence que se construiraient tant bien que mal les identifications et que se jouerait l'accession à la maternité. À défaut, la femme peut rester dans l'ombre d'une mère archaïque dont nul ne viendra la défaire. Ici se loge l'arbitraire du pouvoir maternel tout-puissant lorsqu'il bascule dans le " caprice ". Ici également se nouent les pathologies mère-enfant, à l'endroit où la mère refuse d'apparaître comme déchue, c'est-à-dire comme ne détenant définitivement pas ce que l'enfant désire.

Le cheminement thérapeutique avec ces mères venant nous dire quelque chose de leur souffrance consiste à s'adresser, pourrait-on dire, au narcissisme maternel en restaurant l'enfant qu'elle a été pour qu'elle réhabilite celui qu'elle n'arrive pas à porter, qu'elle porte ou qui vient de naître. Cela implique d'être à l'écoute de ce que la mère nous dit de sa propre rencontre avec 
une imago maternelle. Rencontre que la forme même du discours annonce comme étant soit sous l'emprise de la haine exclusive d'une mère omnipotente ou destructrice, soit sous la fascination de l'amour d'une mère idéalisée.

De plus, au fur et à mesure des étapes de la maternité, l'enfant change de position subjective, et ce mouvement lui fait prendre " un sens » nouveau dans l'économie psychique qui le lie à la mère. De la même façon, ces passages convoquent cette dernière à se déplacer à son tour, et à désinvestir un certain " regard » posé sur l'enfant, pour en investir un autre. Problématique de la perte et du gain que l'on a coutume d'examiner à la lumière des stades du développement de l'enfant, et sur laquelle il me semble essentiel de se pencher du point de vue maternel.

Un nourrisson qui ne parle pas encore ne suscite pas les mêmes remaniements subjectifs chez sa mère qu'un adolescent devenant pubère. Tout commence, nous l'avons vu, par un désinvestissement maternel d'un certain enfant ne pouvant être opérant que s'il fait suite à un temps de "préoccupation maternelle primaire ". Quitter "l'unité narcissique primaire " (Ph. Gutton) qui recouvre ce que d'autres nomment aussi la "dyade" (F. Dolto), repose sur l'hypothèse qu'un CEdipe " précoce " est d'ores et déjà en mouvement, autrement dit, si la mère émerge progressivement de cette phase d' " unité ", c'est en partie dû aux effets de la " castration ombilicale » (Dolto), et d'autre part à l'actuel de ses relations sexuelles.

Puis, après avoir été « objet » de soins (du désir de la mère), l'enfant va devoir se faire une place dans le langage, s'y inscrire, toujours un peu en marge de celle qui lui était assignée dans le fantasme maternel, et ainsi se structurer sur le modèle odipien proprement dit. Ici, de la même façon que l'enfant élabore l'absence de sa mère en se la représentant dans un espace psychique de transition, celui dans lequel ils sont suffisamment proches et suffisamment éloignés, la mère supportera l'absence de son bébé, pouvant, à travers ce désinvestissement, distinguer le champ de la séparation de celui de la mort. Cela est le changement de position subjective maternelle concomitant d'un changement de statut de l'enfant.

C'est ainsi que, progressivement, au rythme de l'adaptation réciproque mère-enfant, l'enfant peut se structurer comme sujet 
autre, entier et sexué dans le fantasme maternel et dans le monde.

On voit bien ici comment ce changement de position subjective chez la mère ne relève pas d'une mise à distance mais d'un déplacement du désir. En changeant d'objet libidinal, la mère retourne à une position génitale. Et s'il ne s'agit pas là à proprement parler d'un " retour ", c'est-à-dire si ce champ était auparavant déserté, le risque demeure de voir l'enfant réduit à occuper une place d'objet réel d'une mère dont la jouissance ne serait pas barrée, c'est-à-dire dont le désir ne serait pas concerné par un autre, et qui viendrait donc se confondre avec la survie de l'enfant.

Dans cette évolution progressive des réaménagements psychiques maternels face à l'enfant qui grandit, le désinvestissement ne saurait succéder qu'à un investissement initial, la désillusion qu'à une illusion fondatrice, le clivage qu'à une unité imaginaire, la haine qu'à l'amour et l'amour qu'à la haine.

Il en est de même lorsque l'enfant est en phase dite de "latence ", qui correspond en fait à cette confrontation avec la loi sociale qu'est l'école. L'entrée à l'école signe socialement la séparation mère-enfant. Nouveau tiers séparateur ne faisant que reconvoquer la fonction tierce paternelle. Pour être mentalement disponible aux apprentissages scolaires, l'enfant doit être en " sécurité psychique ", c'est-à-dire avoir été suffisamment contenu, investi et séparé pour ne pas s'abandonner quand un tiers social le convoque en tant que sujet. En écho, la mère se met à désinvestir le corps de l'enfant dans le rapport aux soins qu'elle lui prodiguait.

Si l'on poursuit sous l'angle de cette psychogenèse, nous voici au temps du pubertaire. L'" œedipe pubertaire " (Ph. Gutton) convoque de nouveau mère et enfant à repenser leurs positions subjectives d'énonciation du désir. Parallèlement au mouvement incestueux que la puberté laisse réémerger, s'instaure pour l'enfant la certitude d'être enfin devenu l'objet adéquat, autrement dit il n'est plus " trop petit ». Et alors, "le pubertaire étant tout l'inverse d'un mouvement de séparation " (Ph. Gutton), voici la mère confrontée à la paradoxale crise d'adolescence où son enfant l'agresse comme s'il voulait vraiment la quitter.

Temps de reformulation de la castration et des interdits, qui nécessite pour la mère de pouvoir entendre cette nouvelle façon 
de poser des questions et de pouvoir en conséquence y apporter une réponse symbolique. Et ce, dans une double remise en question, celle de son statut de mère et celle de son statut de femme. L'adolescence de l'enfant et son versant réel qu'est la puberté, correspondant en général au temps d'un deuil biologique, donc en prise avec le réel, de la procréation pour la mère.

Chacun de ces remaniements réciproques brièvement évoqués reconvoque une symbolisation de la haine revivifiée à chacun de ces temps de passage. Cela pourrait consister en une opposition systématique de tendresse (c'est-à-dire d'amour désérotisé) face à chaque pas vers l'autonomie de l'enfant, c'est-à-dire à chaque rappel de la coupure, et face à l'agressivité pubertaire en particulier, temps de séparation exacerbé.

L'ambivalence maternelle, pour conclure, s'inscrit dans cet entre-deux, entre langage de tendresse et langage de passion. Dans cet espace, c'est parce qu'on aimerait l'enfant qu'on répondrait à sa demande, mais c'est parce qu'on le haïrait qu'on n'y répondrait " pas tout à fait ». Exactement dans cet écart, l'enfant pourra entendre une parole maternelle organisatrice du monde extérieur et aller à la rencontre de ce monde. Contenir l'enfant tout en s'en séparant signe l'ambivalence d'une dynamique maternelle qui consiste à aimer l'enfant pour le contenir, mais aussi à le haïr symboliquement pour s'en séparer. Dans ce langage entre-deux, s'énonce la langue maternelle, celle dans laquelle se déclinent les interdits oedipiens et où s'intriquent le désir et la loi.

Il nous reste à nous, analystes, à entendre la mère qui nous convoque à l'endroit de l'illisibilité de son désir, à en dévoiler les aspects et à en reconnaitre les vacillements qui en font aujourd'hui souvent l'essence. Je pense tout particulièrement aux procréations médicalement assistées et aux souffrances extrêmes dans le registre du social auquel ma pratique de rue me confronte aujourd'hui mais qui fait l'objet d'autres écrits. 